\title{
4. Was können Sie selbst tun, um die Bandbreite Ihrer Kompetenzen zu erweitern?
}

\author{
Eva Lackner, Albert Diefenbacher und Norman Sartorius
}

Nach dem Studium können Sie endlich das angeeignete Wissen im Stationsalltag verantwortlich anwenden und freuen sich sicherlich über Ihre neu gewonnene Selbstständigkeit.

Die erste Arbeitsstelle, gerade in einer psychiatrischen Abteilung, stellt aber auch eine Herausforderung dar und ruft zahlreiche Kompetenzen gleichzeitig ab, die Sie vielleicht noch nicht ausreichend entwickelt haben. Aber was heißt das überhaupt, Optimierung der Bandbreite der persönlichen Kompetenzen? Wie können Sie Ihre „Performance“ entwickeln? Und: Was können Ihre eigenen „Indikatoren“ sein, anhand derer Sie Ihre persönliche Performance einschätzen lernen?

Dazu können zum Beispiel eine erfolgreiche Selbstpräsentation, ein effektives selbstreflexives Handeln und das Erweitern Ihres persönlichen Ressourcenkontingents gehören.

\section{Ausgangslage und Grundhaltung}

An einen Mitarbeiter in einem Krankenhausunternehmen werden zunehmend Anforderungen gestellt, die über die unmittelbar patientenbezogene Arbeit hinausgehen. Qualitätsmanagement, Zertifizierungen, Kennzahlen mit dem Ziel der Vergleichbarkeit und Optimierung von Prozessen sind relevant, und die Steigerung der Effektivität im Vergleich $z u$ anderen Krankenhäusern wird groß geschrieben (Benchmarking). Für viele Vorgänge gibt es Dienst- oder Verfahrensanweisungen, vermutlich auch ein Handbuch, in dem dies alles zusammengefasst ist und das sich mutmaßlich über das Intranet Ihres Krankenhauses abrufen lässt. 
Halt! werden Sie spätestens jetzt rufen. Muss ich als Arzt nicht zuallererst ein guter Diagnostiker sein, um meine Patienten wirkungsvoll behandeln zu können? Stimmt, können wir Ihnen darauf nur antworten, aber mit einer „belastbaren Grundlage“ werden Sie dies umso erfolgreicher bewerkstelligen können!

Welche Grundhaltungen sollten Sie entwickeln?

Jenseits Ihrer spezifischen psychiatrisch-psychotherapeutischen Kompetenz sollten Sie

- ein guter Zuhörer sein,

- empathisch und authentisch sein, dabei aber

- die erforderliche professionelle Distanz aufbringen und

- unterschiedliche kulturelle Kontexte kennen, akzeptieren und berücksichtigen.

Sie sind der Experte! Um dies adäquat zu vermitteln, haben Sie es aber nicht nötig, arrogant zu sein!

Wie Sie die Beziehung zu Ihren Patienten gestalten, wird für Ihren Erfolg wichtig sein: Dabei sollten Sie berücksichtigen, dass in jeder Arzt-Patienten-Beziehung das gemeinsame Entscheiden über Diagnostik- und Therapiemöglichkeiten zum Erreichen einer guten Adhärenz in den Vordergrund zu stellen ist.

Die aktuell gültigen Diagnosesysteme (ICD-10/11, DSM-IV/5) ermöglichen Ihnen ein nach operationalisierten Kriterien vorgegebenes Einordnen des psychopathologischen Befundes in eine „Störungskategorie“. Sie können nach Checklisten Symptome abfragen. Mithilfe des AMDP-Systems ist eine strukturierte psychopathologische Befunderhebung zu Beginn der Ausbildung gut möglich. Ein Vergleich mit Erkrankungsphasen in Voraufenthalten wird ermöglicht, Einschätzungen aus verschiedenen psychiatrischen Abteilungen werden dadurch vergleichbar.

Dabei sollten Sie aber nicht vergessen: Diagnosen sind nur Hilfsmittel! Überspitzt formuliert ist eine Diagnose für den Arzt wichtiger als für den Patienten. Der Patient will wissen, wie er möglichst schnell wieder gesund wird und Sie sollen ihm dabei helfen.

Sie benötigen die Diagnose für Ihre Therapiezielplanung und die von Ihnen im Rahmen der Behandlung auszuwählenden Techniken (bei einer Diagnose aus dem F2-Bereich eher Neuroleptika, bei einer Diagnose aus dem F32-Bereich eher Antidepressiva). Aber der Patient will von Ihnen, dass Sie ihn grundsätzlich unterstützen und ermutigen, z.B. dabei, wie er seine Wohnung behalten und wieder eine Arbeitsstelle bekommen kann. Somit brauchen Sie zusammen mit inm zusätzlich den Kontakt zum Sozialarbeiter und erinnern sich spätestens jetzt daran, dass Sie ein kompetentes multiprofessionelles Team zur Optimierung der erforderlichen Behandlungsstrategien an Ihrer Seite haben. 


\section{Hilfe zur Selbsthilfe - nicht nur für Ihre Patienten wichtig!}

Haben Sie sich für eine psychiatrische Weiterbildung entschieden, befinden Sie sich auf dem Weg in eine sehr erfüllende Tätigkeit. In vielen Fällen können Sie Menschen erfolgreich bei seelischen Krisen begleiten, ihnen wieder Lebensmut geben, sie beim Bewältigen schwerer Erkrankungsphasen unterstützen, ihnen oftmals nach Jahren belastender Ereignisse mit schweren Verlusten und Kränkungen neue Wege aufzeigen.

Einerseits werden Sie dafür Anerkennung erfahren, wenn Sie Ihre „ganz spezielle Profession“ in Gesprächen kundtun, aber es werden auch kritische Reaktionen kommen: Viele Außenstehende, Mitkollegen, vielleicht auch Freunde kommentieren vielleicht: „Ja, Du wirst sehen, wie es Dir im Nachtdienst geht, wenn $\mathrm{Du}$ beschimpft wirst, wenn Du immer wieder erlebst, dass die gerade getroffenen Vereinbarungen nicht eine Minute tragen. Wenn die Patienten nicht kooperieren, ablehnend und aggressiv sind. Solche Disziplin wäre mir $\mathrm{zu}$ anstrengend und nervig!“ oder „Diesen Tätigkeitsbereich würde ich nie aushalten können, diese vielen schrecklichen Schicksale, da kann man ja nie abschalten, da würde ich ja selbst kaputt gehen."

Als Anfänger erleben Sie oft Angst vor Fehlentscheidungen, Unsicherheit im Umgang mit den Patienten und dem multiprofessionellen Team, z.B. in einer Notaufnahme oder auf Station. Es ist wichtig, den Umgang mit Ärger, Enttäuschung, auch den mit der eigenen Hilflosigkeit im Umgang mit Patienten zu reflektieren. Das Ansprechen solcher eigenen Gefühle ist wichtig. Diese können im Rahmen von Gesprächen mit dem Mentor oder mit Kollegen thematisiert werden.

Die interessante Arbeit als Psychiater macht einen sorgsamen Umgang mit den eigenen Ressourcen notwendig.

Stigmatisierung auf verschiedenen Ebenen macht ein stetiges Anpassen der eigenen Kommunikation erforderlich. Es ist sehr wichtig, auch die eigenen Vorurteile zu prüfen. Dabei können z.B. praktische Fragestellungen hilfreich sein, wie: „Würde ich z.B. ein Kindermädchen mit einer überstandenen psychotischen Episode anstellen, würde ich ihr meine Kinder anvertrauen? Wie würde ich mit ihren kritischen Anmerkungen über meinen Erziehungsstil oder mein Verhalten umgehen?" Sie sollten sich gegenüber ehrlich sein, denn nur dann können Sie entsprechende Fragen der Angehörigen und Patienten authentisch beantworten: Mögliche hier auftretende Dilemmata müssen ohnehin die Betroffenen selbst auflösen, diese Entscheidung können Sie ihnen nicht abnehmen. Aber Sie zeigen dadurch, dass Sie die Antworten auf solche Fragen ebenfalls ernst nehmen und sich nicht hinter statistische Wahrscheinlichkeiten und allgemeine Empfehlungen zurückziehen.

Stigmatisierung stellt für viele psychiatrische Patienten eine sehr große Herausforderung dar, führt zu einer Isolation, verhindert einen offenen Umgang mit dem Gesundheitssystem. Notwendige medizinische Hilfen anzunehmen, fällt vielen schwer, da oftmals auch die Entwicklungen im Gesundheitssystem oder bei sozialen Systemen zu wenig barrierefrei sind, sodass Patienten mit psychi- 
schen Problemen oftmals gar nicht medizinisch notwendige Hilfen bekommen oder viel zu spät Hilfe suchen.

\section{Eine ganz besondere Situation - die interdisziplinäre Notaufnahme}

Eine der Besonderheiten in der Psychiatrie ist, dass „auffällig“ gewordene, zum Teil bizarr wirkende, sich in großer Not befindende Patienten unfreiwillig, oft durch die Polizei, vorgestellt werden.

Viele Patienten erleben die Situation in einer Rettungsstelle als große Gefahr, können ihre eigene aktuelle Situation, die eigenen körperlichen Leiden gar nicht wahrnehmen, geschweige denn beschreiben. Oftmals spielen große Schamgefühle eine Rolle. Vorhergehende schlechte Erfahrungen mit dem Gesundheitssystem und auch der Psychiatrie als Helfersystem führen zu einer deutlich ablehnenden Haltung dem unbekannten Arzt gegenüber.

Auch bestehen bei manchen Patienten starke Vorurteile gegenüber einem Arzt, der sich mit dem „Seelenleben“ beschäftigt, sodass die vom Internisten angeregte zusätzliche konsiliarische Beratung in der Notaufnahme eher als „Kränkung“ erlebt wird.

Somit sind diese „ersten Kontaktaufnahmen“ zur Beziehungsgestaltung sehr flexibel zu gestalten und benötigen neben den diagnostischen und therapeutischen Einschätzungen einen empathischen, verständnisvollen und akzeptierenden Kommunikationsstil.

Eine kurze „probatorische“ Einschätzung des Gegenübers ist erforderlich; dabei können zunächst ein offenes Zuhören und Schildern der Bedürfnisse und wenige klärende Fragen hilfreich sein (s. Kap. II.4).

Wegen der vielen gleichzeitigen Anforderungen gerade in den Dienstzeiten besteht für Notfälle ein enges Zeitfenster; das Abrufen effektiver Entscheidungshierarchien und gut in sich greifender diagnostischer Instrumente ist notwendig.

Im Umgang mit dem Behandlungsteam hilft eine klare wechselseitige Kommunikation, ein wertschätzendes, aber auch bestimmendes bzw. abgrenzendes Verhalten.

Im Folgenden soll eine Situation im Bereitschaftsdienst beispielhaft skizziert werden.

Ein mit Alkohol intoxikierter Patient wird von der Polizei in die Rettungsstelle eingeliefert, ist laut und aggressiv, kündigt an: „Es ist mir egal, was aus meinem Leben wird, für mich hat sich eh noch nie jemand interessiert!". Im selben Moment wird ein anderer Patient fixiert von Polizei und Feuerwehr gebracht, der erregt und wild gestikulierend auf der Straße gestanden und versucht habe, den Verkehr zu regeln, „komisches Zeug und verwirrt“ rede, aber auch „komisch zittere und eine Verletzung am Bein habe, die echt nicht gut aussieht“. Eine der psychiatrischen Stationen meldet sich parallel mit dem sofortigen Entlassungswunsch eines Patienten, mit dem Sie nun umgehend sprechen sollen. Ein anderer Patient auf einer gerontopsychiatrischen Station sei gestürzt, nachdem er schon den ganzen Tag auffällige Blutzuckerwerte gehabt habe. Wenige Minuten später kommt auch noch der Internist und gibt an, er sei fertig mit dem älteren Herrn in Raum 5, aus seiner Sicht sei er nicht internistisch behandlungsbedürftig, die Ehefrau wolle aber unbedingt, dass er aufgenommen werde. Die Situation sei „anstrengend" und mache eine rasche Klärung, am besten die Aufnahme in die Psychiatrie, erforderlich. 


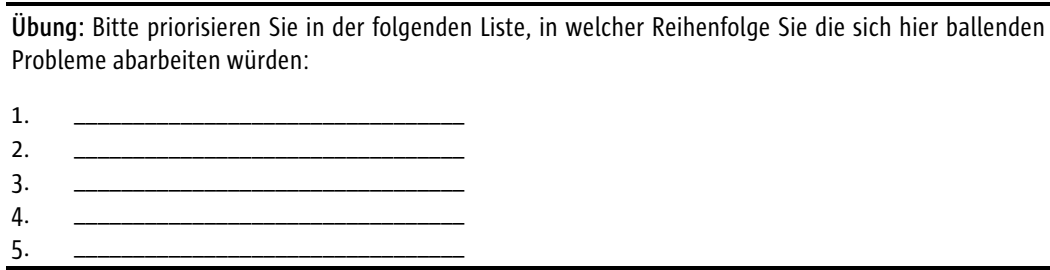

Eine „objektive“ quasi-verbindliche Reihenfolge gibt es nicht: Sie müssen unter Berücksichtigung der aktuellen Gegebenheiten entscheiden. Wichtig ist nur: Sie müssen entscheiden!

Oftmals ist man mit den Kommunikationsstrategien der anderen „Mitstreiter“ nicht einverstanden, was zunächst die eigene Zurücknahme bzw. Kompromissbereitschaft erfordern kann, aber auch Mut notwendig macht, um Grenzen und Korrekturen ermöglichen zu können, damit der Patient die bestmögliche notwendige Behandlung bekommt.

Einfach zu klärende Rückfragen sind notwendig, um einen guten Ablauf gewährleisten zu können. Parallel dazu machen oftmals die angespannte Crundstimmung gerade in einer Notaufnahme und der Zeitdruck der Polizei oder Feuerwehr ein ruhiges Abarbeiten schwer. Oftmals erfordert es Mut, sich erfahreneren Kollegen anderer Fachrichtungen gegenüber abzugrenzen, z.B. auch ein Übernahmeanliegen stringent abzulehnen, oder dem Pflegepersonal klare Aufträge zu erteilen.

Das erfordert Standhaftigkeit! Die eigene Kompromissbereitschaft führt oft, leider aber nicht immer, zu entsprechender Kompromissbereitschaft des Gegenübers.

Somatische Komorbiditäten spielen bei Patienten mit psychischen Erkrankungen eine große Rolle und machen in jedem Fall eine gute Zusammenarbeit mit den somatischen Kollegen erforderlich. Deren zum Teil stigmatisierende Haltung zu überwinden, ist manchmal schwierig; ebenso sorgen Psychiater mit ihren eigenen Einschätzungen durchaus auch beim einen oder anderen Kollegen für Kopfschütteln (s. Kap. II.5).

Eine gute Kompetenz in der körperlichen und speziell neurologischen Untersuchung ist daher wichtig, fundierte Kenntnisse in den somatischen Fächern und das Abrufen wichtiger Symptome körperlicher Erkrankungen erleichtern die differenzialdiagnostische Einordnung und sichern die richtige therapeutische Entscheidung und das Kommunizieren mit den somatischen Kollegen. Gerade in unklaren „somato-psychischen Krisensituationen“, wenn eine gemeinsame Besprechung aktueller Laborwerte bzw. bildgebender Befunde erfolgen sollte, hilft diese Basis, um vorschnelle Aufnahmen verhaltensauffälliger Patienten auf eine psychiatrische Station zu vermeiden, insbesondere, wenn dort keine ausreichende Überwachungskompetenz vorhanden ist (s. Kap. II.5 u. IV.1)!

Der Umgang mit einem Menschen, der akut gefährdet und nicht „absprachefähig“ ist, macht ein klares, sicheres Vorgehen unter Berücksichtigung der rechtlichen Grundlagen zum Teil gegen den Willen des Patienten erforderlich. Eine en- 
ge Zusammenarbeit mit den jeweils zuständigen Teams ist notwendig. Entscheidungen über Medikamente müssen akut und sicher gefällt werden, die Einordnung des Intoxikationsgrades muss mit den anderen Fachrichtungen bzw. dem Giftnotruf besprochen werden. Überwachungszeiträume und entsprechende Zuständigkeiten müssen geklärt werden, bevor eine Aufnahme in den psychiatrischen Bereich erfolgen kann.

Es ist sehr hilfreich, Familienangehörige oder enge Freunde/Bezugspersonen zu fragen, welche erprobten „Kommandos, Verhaltensrichtlinien oder Kommunikations-Leitsätze“ im Umgang mit dem Patienten z.B. im Falle eines Erregungszustandes wirksam sind. Die Zusammenarbeit mit Angehörigen erweist sich oft als förderlich. Diesen hilfreiche Kommunikations- und Verhaltenshinweise zu erläutern, wirkt oft sehr unterstützend. Weiterhin können gemeinsam mit dem Patienten und dem persönlichen Umfeld des Patienten Behandlungsvereinbarungen für eventuelle weitere Krisen erarbeitet werden. Gemeinsam gestaltete Notfallpläne mit u.a. hilfreichen Medikamenten unterstützen das Krisenmanagement.

\section{Wie kann eine Unterstützung aussehen?}

Wir haben in unserer Abteilung für Psychiatrie, Psychotherapie und Psychosomatik im Ev. Krankenhaus Königin Elisabeth Herzberge in Berlin folgende Bausteine gemeinsam mit unseren Assistenzärzten entwickelt, die sich unserer Einschätzung nach für die Entwicklung einer guten Performance bewährt haben:

- Ein Mentor (erfahrener AIW, FA oder OA) kümmert sich um den Berufsanfänger und kann den Einarbeitungsprozess über einen längeren Zeitraum begleiten.

- In einem obligatorischen „Basiscurriculum“ werden allen neuen ärztlichen Mitarbeitern Grundkenntnisse zu wichtigen Themen nahegebracht: Suizidalität, rechtliche Grundlagen im Umgang mit Zwangsmaßnahmen (Psych-KG, Betreuungsgesetz), Notfallsituationen in der Psychiatrie, psychopathologische Befunderhebung, ambulante und teilstationäre Behandlungsmöglichkeiten, Komplementäreinrichtungen im eigenen Versorgungsbezirk.

- Zusätzlich ist neben einem wöchentlichen Journal-Club die Teilnahme an einem strukturierten Fortbildungsprogramm mit wöchentlichen berufsgruppenübergreifenden Fortbildungen mit unterschiedlichen Schwerpunkten möglich.

- „Beginner-Workshops“, an denen auch Assistenzärzte anderer psychiatrischer Abteilungen teilnehmen können, bereiten durch kasuistische Präsentationen und Kleingruppenarbeit zu den Themen Psychopathologischer Befund, Behandlung von Notfällen, Umgang mit Neuroleptika und anderen Psychopharmaka, aber auch Zeitmanagement auf den psychiatrischen Alltag vor.

- AMDP-Kurse und regelmäßige AMDP-Fortbildungen mit Beispielen eigener Patienten und Videoaufnahmen verdeutlichen praktisch die einzelnen psychopathologischen Syndrome. Das AMDP-System-Manual und der strukturierte Fragekatalog helfen, effektive Fragen zu stellen, und erläutern detailliert die symptomausprägungen. 
- Eine sichere Routine bei der orientierenden neurologischen (und internistischen) Untersuchung ist sehr hilfreich (s. Kap. IV.1).

- Haben Sie sich in der Erhebung des strukturierten psychopathologischen Befundes erst einmal „freigeschwommen“, sollten Sie etwa einige Arbeiten des Psychiaters Christian Scharfetter (1936-2012) lesen, um einen erweiterten Blick für verschiedene Untereinheiten des Denkens und Fühlens von Menschen z.B. mit schizophrenen Erkrankungen zu gewinnen (Scharfetter 1995).

\section{Gesprächsführungstechniken und weiteres „Rüstzeug“ für Ihre Ausbildung}

- Breaking Bad News-Kurse werden z.B. von der Ärztekammer angeboten. Das Überbringen schlechter Nachrichten gehört zu den am meisten ungeliebten und gefürchteten Aufgaben im Krankenhaus. Dabei kann diese Form der Kommunikation erlernt werden. Der Verlauf eines solchen Gespräches hat nichts mit Empathie oder Gespür zu tun, sondern mit professionellem Handeln und einer kontrollierten Gesprächsführung (Langkafel 2008).

- Motivational Interviewing: Diese Technik beinhaltet sowohl einen Klienten zentrierten als auch direktiven Ansatz der Gesprächsführung zur Erhöhung der Eigenmotivation von Menschen, problematisches Verhalten, z.B. Suchtmittelabusus, zu ändern (Miller et al. 2009).

- Shared decision making (SDM) ist eine spezifische Form der Interaktion zwischen medizinischem Personal und Patient, welche auf geteilter Information und gleichberechtigter Entscheidungsfindung z.B. bei Themen bezüglich der Diagnose und Therapieentwicklung basiert. Um den Prozess dieser „Partizipativen Entscheidungsfindung“ $\mathrm{zu}$ verstehen und $\mathrm{zu}$ erlernen, sind Workshops sehr hilfreich.

- Deeskalationsseminare vertiefen und beleuchten mit verschiedenen Berufsgruppen schwierige Situationen in der Psychiatrie, u.a. das Thema Zwangsmaßnahmen, aus verschiedenen Perspektiven. Sie eröffnen Strategien, solche Situationen zu verhindern, und ermöglichen das praktische Üben (s. Kap. I.8).

Schreiben Sie ein „Tagebuch Ihrer eigenen Weiterbildung“!

- Während der psychiatrischen Ausbildung ist das Führen eines Tagesbuches eine gute Unterstützung, um eigene kritische Situationen, persönliche „Meilensteine“, hilfreiche „Tools“ festzuhalten.

- Sie könnten problematische Situationen oder Empfindungen, die Sie im klinischen Alltag belasten, in dem Tagebuch aufschreiben. Wichtig dabei: Sie notieren, was Ihnen geholfen hat, mit den Belastungen fertig zu werden! Sie werden sehen, nach einiger Zeit gewinnen Sie hierdurch an Selbstvertrauen. Ihre eigenen Fortschritte können Sie so nachhaltig mitverfolgen.

- Möglich ist aber auch: Sie merken, dass Sie mit Einigem, was regelhaft auf Sie zukommt, nicht in für Sie befriedigender Weise umgehen können oder umgehen wollen. Über ein solches Tagebuch können Sie wichtige persönliche Erkenntnisse und Gefühle gegenüber Ihrer Profession sehr gut nachspüren und sich diesbezüglich selbstkritisch reflektieren.

- Auch wenn es Überwindung kostet: Manchmal ist eine Neuorientierung bzw. „kleine Korrektur“, was den weiteren beruflichen Karriereweg angeht, eine wichtige Erkenntnis. Es gibt keinen Grund, dass Sie bei Ihrer Arbeit freiwillig leiden! 
- Zeitmanagement-Kurse ermöglichen eine effektive Arbeitsorganisation bezogen auf die Tagesplanung, „Zeitdiebe“ können in Selbstanalyse erfasst werden, Prioritäten setzen und Delegieren erleichtern die Arbeitsabläufe (s. Kap. II.1).

- Die Auseinandersetzung mit der eigenen Arztrolle (s. Kap. I.1 u. I.2) und der eigenen persönlichen Repräsentation hilft beim Entwickeln bzw. Anpassen der „eigenen Ausstrahlung/Haltung“.

- Regelmäßige Gespräche („Protected hours“) mit dem zuständigen Ober- bzw. Facharzt ermöglichen den fachlichen Austausch. Positive, aber auch kritische Rückmeldungen auf beiden Seiten stärken die kommunikativen Fertigkeiten und das Selbstbild. Das aktive Einfordern und offene Ansprechen von Problemfeldern/Bedürfnissen erleichtert die Zusammenarbeit und die eigene Weiterentwicklung.

Sie erwarten ja von Ihrem Klavier- oder Tennislehrer auch, dass er Sie, in gebührender Form, auf Ihr Verbesserungspotenzial aufmerksam macht, also Ihre Fehler benennt und korrigiert.

- Schwierige Gesprächssituationen in Psychiatrie und Psychotherapie werden in einem Buch von Jacob et al. (2009) dargestellt. Auch der Umgang mit einem Suizid in der Klinik wird darin als Beispiel thematisiert: Die kurze und sachliche Information der Patienten auf einer Stationsversammlung ist wichtig, Information der Angehörigen notwendig. Einzelgespräche mit allen Patienten sind zu empfehlen. Des Weiteren ist ein vertrauensvolles Gespräch mit dem Vorgesetzten und dem Team sehr unterstützend. Eine Fallaufarbeitung aus den verschiedenen Perspektiven der therapeutischen Begleitpersonen der Betroffenen im Rahmen einer Fortbildung vertieft die Problematik.

- Rollenspiele schulen die Wahrnehmung, üben den Umgang mit beispielhaften Alltagssituationen und ermöglichen das Beleuchten aus unterschiedlichen Blickwinkeln: Hier ist insbesondere auch die Trio-Technik zu empfehlen, ein englisches Konzept von Gask/Goldberg (2008).

\section{Triotechnik}

Gruppen finden sich zu dritt zusammen und üben Beispielsituationen, wie den Umgang mit Diagnoseübermittlung oder das Thema Medikamenteneinnahme an alltagsüblichen Fällen: z.B. Patient lehnt Medikation $a b$. Diese Situation wird in folgender Konstellation durchgespielt: Einer ist Patient, der andere ist der Arzt, die dritte Person fungiert als Beobachter der Situation. Jede der Rollen wird getauscht. Im Vorfeld werden entsprechende Tools für die Kommunikation (Feedbackregeln, Vorgeben der Symptome, genaue Vorinformation an den Arzt- bzw. Patienten-Part) durchgesprochen, Haltungen und wichtige Lernziele erläutert. Solche Trainingseinheiten helfen beim „Sicherwerden“ für den Ernstfall in der Rettungsstelle oder auf Station.

- Die Teilnahme an Balintgruppen oder, im Rahmen eines verhaltenstherapeutischen Curriculums, an sog. IFA-Gruppen (interaktionelle Fallarbeit) ermöglicht zusätzlich den gegenseitigen Austausch. Das Besprechen besonderer Fälle schult die Reflexion aus unterschiedlichen Blickwinkeln. Entscheiden Sie vorab, ob es für Sie wichtig ist, dass der Supervisor, mit dem Sie zusammenarbeiten wollen, als solcher zertifiziert ist. 
- Das AMSP-Projekt dokumentiert und analysiert seit 1993 systematisch unerwünschte Arzneimittelwirkungen von Psychopharmaka mit dem Ziel, die Behandlungssicherheit durch Dokumentation und Weiterbildung zu erhöhen. In Berlin arbeiten verschiedene Kliniken zusammen. Das regelmäßige, ritualisierte Ansprechen von Nebenwirkungen, zum Beispiel in unserer Abteilung während der sog. „Mittagsrunde“, schult gegenseitig die Wahrnehmung und Aufmerksamkeit. Die Falldokumentationen ermöglichen einen nationalen und internationalen Austausch bzgl. schwerer UAW von Psychopharmaka. Die eigene Suche nach Wechselwirkungen verschiedener Medikamente wird über Suchinstrumente wie zum Beispiel MediQ gefestigt und schult den Austausch mit anderen Fachrichtungen. Anmeldung über die Website von MediQ unter https://www.mediq.ch/.

- Der offene Umgang mit Fehlern im Stationsalltag (kritische Vorkommnisse und Beinahefehler) wird über das wichtige Bericht- oder Melde-System CIRS (Critical Incident Reporting System) unterstützt (s. Kap. I.6).

- Wir halten den intensiven Erfahrungsaustausch mit Angehörigen und Betroffenen außerhalb des eigenen Krankenhauses für wichtig. Die aktive Teilnahme an der Angehörigenarbeit schult den Blickwechsel, übt den Umgang mit eigenen Grenzen und ermöglicht einen vielschichtigen Einblick in die Alltagsrelevanz vieler seelischer Erkrankungen.

Zusammenarbeit mit Betroffenen und Angehörigen

- Besuch von Veranstaltungen der lokalen Angehörigenverbände, jeweilige Landesinfo unter dem Bundesverband der Angehörigen psychisch Kranker (BApK, Selbsthilfeorganisation und Solidargemeinschaft von Familien mit psychisch Kranken), siehe auch unter der Website: http:// www.psychiatrie.de/bapk/top/kontakt/lvs/, lokal in Berlin: http://www.apk-berlin.de/

- http://www.sunnysideup-berlin.de/, eine hilfreiche Seite für Kinder psychisch erkrankter Eltern

- Teilnahme an trialogischen Seminaren (in Berlin z.B. von Albatros, jeweils lokal in den Bundesländern unter Trialog Psychoseseminar abrufbar)

- Besuch von komplementären sozialpsychiatrischen Einrichtungen in der eigenen Versorgungsregion, Pinel, http://www.pinel-online.de/

- Netzwerk Stimmenhören, siehe auch unter: http://www.stimmenhoeren.de/

- http://www.psychiatrie.de/, allgemeine Informationen

- Gemeinsame Treffen, z.B. mit der Polizei, üben den Umgang mit den richtigen Begrifflichkeiten beim Einfordern von Unterstützung. Sie führen zum kritischen Austausch über gemeinsame Problemfälle und erleichtern dadurch die konstruktive Zusammenarbeit (s. Kap. III.5).

\section{Vernetzung}

Sie haben viele Möglichkeiten, sich lokal, regional oder national zu vernetzen: Tauschen Sie sich einfach mit Ihren Kolleginnen und Kollegen aus! Wir möchten hierfür eine Empfehlung geben: Lesen Sie das Kapitel von Cécile Hanon et al. in diesem Buch (s. Kap. V.6) und schauen Sie einmal nach, was die Section of Young Psychiatrists der European Psychiatric Association macht (www.europsy.net/). 
Vergessen Sie auch nicht die Vernetzung mit Ärzten anderer Fachrichtungen: Informieren Sie sich über die Beiträge, die Sie als Psychiater im Konzert der medizinischen Fächer beisteuern können (z.B. Ferrari et al., in Druck).

\section{Literaturempfehlung}

AMDP, Arbeitsgemeinschaft für Methodik und Dokumentation in der Psychiatrie (2007) Das AMDP-System. Manual zur Dokumentation psychiatrischer Befunde. 8. Aufl. Hogrefe Göttingen

Berger M (Hrsg.) (2015) Psychische Erkrankungen. Klinik und Therapie. 5. Aufl. Urban \& Fischer in Elsevier München. Gesamtwerk mit einer guten Übersicht über alle wichtigen Themen der psychiatrischen Weiterbildung und online verfügbaren Aktualisierungen.

Berg-Peer I (2013) Schizophrenie ist scheiße, Mama! Vom Leben mit meiner psychisch erkrankten Tochter. Fischer Taschenbuch Frankfurt/Main

Burian R, Diefenbacher A (2011) Arzneimittelsicherheit in der Psychiatrie. Ist das Pharmakovigilanzprogramm von der Klinik in den ambulanten Sektor übertragbar? Soziale Psychiatrie 35(2), 43-45

Demmel R (2012) Motivational Interviewing. Lautes Nachdenken über Veränderung. Psychotherapeutenjournal 100-104

Diefenbacher A, Saupe R (Hrsg.) (1996) Praktische Konsiliarpsychiatrie und -psychiatrie. Enke Stuttgart. Hier werden anhand von praktischen Beispielen wichtige konsiliarische Fragen, die sich dem Anfänger etwa im Bereitschaftsdienst stellen, anschaulich bearbeitet. (leider vergriffen und nur über Bibliotheken erhältlich aber immer noch lesenswert!)

Dörner K, Plog U, Teller C (2010) Irren ist menschlich. Lehrbuch der Psychiatrie/Psychotherapie. 5. Aufl. Psychiatrie-Verlag Köln. „Irren ist menschlich“ ist seit über 30 Jahren das sozialpsychiatrische Standardwerk. Das Buch thematisiert insbesondere die Haltung, wie wir alle uns psychischen Beeinträchtigungen und Krankheiten annähern. Dadurch wird das Verständnis für die Krankheitsbilder und die betroffenen Menschen näher gebracht.

Fähndrich E, Stieglitz R-D (2007) Leitfaden zur Erfassung des psychopathologischen Befundes. Halbstrukturiertes Interview anhand des AMDP-Systems. 3. Aufl. Hogrefe Göttingen. Sehr hilfreich für den Anfänger sind die Vorschläge, wie die einzelnen psychopathologischen Symptome in der Exploration zu erfragen sind.

Ferrari S, Mattei D, Dreher A, Diefenbacher A (in Druck): Liaison psychiatry: is it possible. In: Fiorillo A, Volpe U, Bhugra D (Hrsg.) Psychiatry in practice. A guide for Early Career Psychiatrists. Oxford University Press Oxford

Gask L, Goldberg D (2008) Methods of training and education about depression. Editors of the educational programme: Mario Maj, Norman Sartorius, Allan Tasman and Oye Gureje. URL: http://www.wpanet.org/ uploads/Education/Educational_Programs/depressive-disorders-volume4.pdf (abgerufen am 27.01.2015)

Jacob G, Lieb K, Berger M, Normann C, Jähne A (Hrsg.) (2009) Schwierige Gesprächssituationen in Psychiatrie und Psychotherapie. Elsevier/Urban \& Fischer München

Klinger C, Diefenbacher A (2008) Arzneimittelsicherhet in der Psychiatrie. Psychosoziale Umschau 4, 29-30

Kuiper PC, Menges M (1995) Seelenfinsternis. Die Depression eines Psychiaters. (Geist und Psyche, 12764). Fischer Frankfurt am Main

Langkafel P (2008) Breaking Bad News. Das Überbringen schlechter Nachrichten in der Medizin. (Gesundheitswesen in der Praxis). Economica Heidelberg

Lauveng A (2010) Morgen bin ich ein Löwe. Wie ich die Schizophrenie besiegte. btb München

Miller WR, Rollnick S (2009) Motivierende Gesprächsführung. 3. Aufl. Lambertus Freiburg

Scharfetter C (1995) Schizophrene Menschen. Diagnostik, Psychopathologie, Forschungsansätze. 4. Aufl. Beltz, PsychologieVerlagsUnion Weinheim. Hier finden Sie eine einführende und umfassende Darstellung zum Verständnis schizophrener Menschen. Im Mittelpunkt der Betrachtungen stehen das unmittelbare Erscheinungsbild der Störung selbst und das Erleben des Patienten.

Scharfetter C (2010) Allgemeine Psychopathologie. Eine Einführung. 6. Aufl. Thieme Stuttgart

Sekeres MA, Stern TA (Hrsg.) On the edge of life. Diary of a medical intensive care unit. MGH Psychiatry Academy Boston, MA

The Royal College of Psychiatrists (2014) The National Schizophrenia Fellowship on treatment guidelines. Unter Mitarbeit von Gary Hogman. URL: http://pb.rcpsych.org/content/25/8/289.full (abgerufen am 27.01.2015). Angehörige haben an diesen Empfehlungen entscheidend mitgearbeitet. 
I Ärztliche Haltung im Alltag - praktische Tipps

Voderholzer U, Hohagen F (Hrsg.) (2014) Therapie psychischer Erkrankungen. 10. Aufl. Urban \& Fischer in Elsevier München. Dieses Buch thematisiert in seinen Kapiteln die aktuellen evidenzbasierten Therapieempfehlungen psychiatrischer Erkrankungen. Sie basieren u.a. auf den STATE OF THE ART Symposien des DGPPN-Kongresses des jeweiligen Jahres.

Wienberg G, Berg M, Walther C (2013) PEGASUS. Psychoedukative Gruppenarbeit mit schizophren und schizoaffektiv erkrankten Menschen. Psychosoziale Arbeitshilfen, 30. 6. Aufl. Psychiatrie Verlag Köln

Yalom ID (2002) Der Panama-Hut oder was einen guten Therapeuten ausmacht. Goldmann München. Wie sieht es aus, das richtige Verhältnis zwischen Therapeut und Klient? Nach über 50 Jahren Arbeiten in einem spannenden Arbeitsfeld beschreibt der Autor wichtige und lehrreiche Situationen in Therapiesitzungen und gibt dabei viele hilfreiche Tipps.

\section{Wissenschaftliche Zeitschriften, deren regelmäßige Lektüre wir Ihnen empfehlen möchten}

Current Opinion in psychiatry: http://journals.lww.com/co-psychiatry/pages/default.aspx

Evidence based mental health: http://ebmh.bmj.com/

World Psychiatry: http://www.world-psychiatry.com/ 